ISSN 2413-0877 Volume 2 (2015) 363

The 3rd International Conference on Biological Science 2013

(The 3rd ICBS-2013)

\title{
LIVE IMAGING OF ERK ACTIVITY STEPWISE PATTERNING DURING SOMITOGENES
}

\author{
Dini Wahyu Kartika Sari, Ryutaro Akiyama, Takaaki Matsui, and Yasumasa Bessho \\ Gene Expression Research Laboratory, Graduate School of Biological Sciences, \\ Nara Institute of Science and Technology
}

\begin{abstract}
Periodical segmentation of the anterior extremity of the presomitic mesoderm (PSM) generates metameric structure of somites during vertebrate development. During somite segmentation in zebrafish, msep determines a future somite boundary at position B-2 within the PSM. However, heat shock experiments suggest that an earlier future of somite boundary exists at B-5, but the molecular signature of this boundary remains unidentified. Our recent study demonstrated that fibroblast growth factor (FGF) gradient is converted into an ON-OFF boundary of downstream Erk activity, which corresponds to the future B-5 somite boundary. Moreover, we also revealed that the segmentation clock is required for a stepwise posterior shift of the Erk activity boundary during each segmentation. To clarify this evidence, here we perform time-lapse imaging of Erk activity in living embryos using a FRET biosensor. We focused on FRET signals within the PSM to observe spatial and temporal changes of Erk activity. Consistent with the data from fixed embryos, we observed ON-OFF boundary of Erk activity within the PSM and the position stepwisely shifted to posterior during somite formation. In order to test the contribution of the segmentation clock to the stepwise movements of the ON-OFF boundary of Erk activity, we disrupted the segmentation clock in zebrafish embryos by knocking down two segmentation clock genes, her1 and her7. Double knockdown of her1 and her7 resulted in the failure of the shift of the Erk ON-OFF boundary during somite segmentation, leading to segmentation defects of somites. These results strongly suggest that the clock-dependent stepwise movement of Erk activity is a key mechanism to generate the perfect repetitive structure of somites.
\end{abstract}

Key words: Live imaging, Erk, stepwise, somitogenesis

ISSN 2413-0877 (C) 2015 The Authors.

Published by KnowledgeE Publishing Services This is an open access article under the CC BY-NC-ND license (http://creativecommons.org/licenses/by-nc-nd/4.0)

Selection and Peer-review under responsibility of the 3rd ICBS-2013

Doi http://dx.doi.org/10.18502/kls.v2i1.176 